\title{
Cardiovascular disease in women with breast cancer - a nationwide cohort study
}

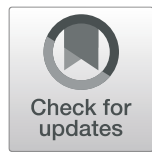

Marie Jakobsen ${ }^{1 *} \mathbb{D}$, Christophe Kolodziejczyk1, Morten Sall Jensen², Peter Bo Poulsen³, Humma Khan³, Thomas Kümler ${ }^{4}$ and Michael Andersson ${ }^{5}$

\begin{abstract}
Background: There is increasing concern about cardiovascular disease (CVD) after breast cancer (BC). The aim of this study was to estimate the prevalence of different types of CVD in women diagnosed with $B C$ compared to cancer-free controls as well as the incidence of CVD after BC diagnosis.
\end{abstract}

Methods: We performed a cohort study based on data from national registries covering the entire Danish population. We followed 16,505 cancer-naive BC patients diagnosed from 2003 to 20075 years before and up to 10 years after BC diagnosis compared to 165,042 cancer-free controls.

Results: We found that $15.6 \%$ of $B C$ patients were registered with at least one CVD diagnosis in hospital records before BC diagnosis. Overall, BC patients and controls were similar with regard to CVD comorbidity before BC diagnosis. After $B C$ diagnosis, the incidence of all CVD diagnoses combined was significantly higher in $B C$ patients than controls up to approximately 6 years after the index date (BC diagnosis). After 10 years, $28 \%$ of both BC patients and controls (without any CVD diagnosis up to 5 years before the index date) had at least one CVD diagnosis according to hospital records. However, the incidence of heart failure, thrombophlebitis/thrombosis and pulmonary heart disease including pulmonary embolism remained higher in BC patients than controls during the entire 10-year follow-up period. After 10 years, $2.7 \%$ of BC patients compared to $2.5 \%$ of controls were diagnosed with heart failure, $2.7 \%$ of $\mathrm{BC}$ patients compared to $1.5 \%$ of controls were diagnosed with thrombophlebitis/ thrombosis, and $1.5 \%$ of $\mathrm{BC}$ patients compared to $1.0 \%$ of controls were diagnosed with pulmonary heart disease according to hospital records. Furthermore, we found that the risk of heart failure and thrombophlebitis/thrombosis was higher after chemotherapy.

Conclusions: Focus on CVD in BC patients is important to ensure optimum treatment with regard to $B C$ as well as possible CVD. Strategies to minimise and manage the increased risk of heart failure, thrombophlebitis/thrombosis and pulmonary heart disease including pulmonary embolism in BC patients are especially important.

Keywords: Breast cancer, Cardiovascular disease, Prevalence, Incidence, Cohort study, Matched control group

\footnotetext{
* Correspondence: mariejakobsen@yahoo.dk

'VIVE, The Danish Center for Social Science Research, Herluf Trolles Gade 11, DK-1152 Copenhagen K, Denmark

Full list of author information is available at the end of the article
}

(c) The Author(s). 2021 Open Access This article is licensed under a Creative Commons Attribution 4.0 International License, which permits use, sharing, adaptation, distribution and reproduction in any medium or format, as long as you give appropriate credit to the original author(s) and the source, provide a link to the Creative Commons licence, and indicate if changes were made. The images or other third party material in this article are included in the article's Creative Commons licence, unless indicated otherwise in a credit line to the material. If material is not included in the article's Creative Commons licence and your intended use is not permitted by statutory regulation or exceeds the permitted use, you will need to obtain permission directly from the copyright holder. To view a copy of this licence, visit http://creativecommons.org/licenses/by/4.0/. The Creative Commons Public Domain Dedication waiver (http://creativecommons.org/publicdomain/zero/1.0/) applies to the data made available in this article, unless otherwise stated in a credit line to the data. 


\section{Background}

Breast cancer $(\mathrm{BC})$ is the most prevalent type of cancer among women worldwide (https:/gco.iarc.fr/today/data/factsheets/ populations/900-world-fact-sheets.pdf). In Denmark, approximately 4700 women are diagnosed with BC every year, corresponding to $25 \%$ of all new primary cancer cases among women, and $10 \%$ of all women are diagnosed with $\mathrm{BC}$ before they become 75 years old (http://www-dep.iarc.fr/NORDCAN/ DK/frame.asp).

Many patients have coexisting diseases (comorbidity) when they are diagnosed with cancer, especially elderly cancer patients [1]. International studies show that 20$35 \%$ of $\mathrm{BC}$ patients have one or more comorbidities at the time of $\mathrm{BC}$ diagnosis [2]. In Danish women diagnosed with early stage $\mathrm{BC}$, the percentage with comorbidity has been shown to be approximately $20 \%[3,4]$.

Most existing studies assess comorbidity in BC patients using an aggregated measure such as the Charlson Comorbidity Index (CCI) [2-7]. Fewer studies provide information on the prevalence and incidence of specific comorbid conditions and different types of CVD in particular [8-10]. Such information is critical to inform clinicians and ensure optimum treatment of $\mathrm{BC}$ patients with regard to $\mathrm{BC}$ as well as possible CVD.

Abdel-Qadir et al. [9] have shown that women diagnosed with early stage $\mathrm{BC}$ are more likely to have a history of heart failure, ischemic heart disease, cerebrovascular disease, peripheral vascular disease, atrial fibrillation and hypertension compared to cancer-free controls, and that the incidence of heart failure, cerebrovascular disease and arrhythmias is significantly higher in $\mathrm{BC}$ patients up to 10 years after BC diagnosis. Strongman et al. [10] find that the incidence of heart failure, venous thromboembolism and pericarditis is significantly higher in $\mathrm{BC}$ survivors compared to cancer-free controls up to 2 years after BC diagnosis, but do not find a higher incidence of stroke or arrhythmias as in the study by Abdel-Qadir et al. Both studies are based on registry data covering a segment of the population in Canada and the UK, respectively. Even though the segment of the UK population (6.9\%) in the study by Strongman et al. has been shown to be broadly representative of the general UK population in terms of age, gender and ethnicity [11], a risk of selection bias remains as the study population can differ from the general population in other ways.

The overall aim of our study was to estimate the prevalence and incidence of different types of CVD in $\mathrm{BC}$ patients compared to cancer-free controls up to 5 years before and 10 years after $\mathrm{BC}$ diagnosis based on registry data covering the entire Danish population. Furthermore, we investigate the risk of developing CVD in $\mathrm{BC}$ patients following chemotherapy, radiation therapy, antibody therapy and hormonal therapy. Compared with previous studies, our study includes a larger and less segmented study population of BC patients and a more precisely matched control group.

\section{Methods}

We performed a cohort study based on data from national registries covering the entire Danish population (5.8 million inhabitants). Data from the Danish Cancer Registry (CAR) [12], the Danish National Patient Registry (NPR) [13, 14], the Danish National Prescription Registry for Drugs (NPRD) [15, 16], Danish education registers [17] and the Danish Civil Registration System [18] were linked using the unique personal identification number assigned to every citizen in Denmark (known as the CPR number).

\section{Study population}

We used CAR to identify all women who were $18+$ years old and diagnosed with BC in Denmark during the period from 1 January 2003 to 31 December 2007. CAR is a research registry containing information at the individual level on the incidence of cancer in the Danish population since 1943 [12]. Diagnoses in CAR are coded according to the International Classification of Diseases 7th Revision (ICD-7) from 1943 to 1978 and according to the International Classification of Diseases 10th Revision (ICD-10) from 1978 and onwards [12]. We used the diagnosis code $\mathrm{C} 50 *$ (ICD-10) to identify $\mathrm{BC}$ patients in the study population. Women who were registered in CAR with any cancer diagnosis (ICD-10: $\mathrm{C00*}$-C97* or ICD-7: $\left.140 *-207^{*}\right)$ prior to the BC diagnosis or who had lived outside Denmark for more than 1 year when they were $18+$ years old were excluded to allow focus on newly diagnosed $\mathrm{BC}$ patients who had no previous cancer diagnosis (cancer-naïve $\mathrm{BC}$ patients). The index date was that of $\mathrm{BC}$ diagnosis in CAR.

Subgroups of $\mathrm{BC}$ patients were defined according to age group $(<45,45-74$ and $75+)$ and cancer disease stage (stage I-III, stage IV or unknown) at index date. Data on cancer disease stage at index date were obtained from CAR.

Exact matching was used to identify a control group of women from the general population in Denmark without any cancer diagnosis in CAR (ICD-10: $\mathrm{C} 00^{*}-\mathrm{C} 97^{*}$ or ICD-7: $140^{*}-207^{*}$ ) up to and including the index year (i.e. the year of $\mathrm{BC}$ diagnosis of the matched $\mathrm{BC}$ patient). For each $\mathrm{BC}$ patient, we identified 10 controls matched according to age group $(<30,30-34,35-39,40-44,45-$ 49, 50-54, 55-59, 60-64, 65-69, 70-74, 75-80, 80-85, $85+$ ), region of residence (Capital Region, Region Zealand, Region of Southern Denmark, Central Jutland Region, North Jutland Region) and education (low, medium, high, unknown). Each person in the control 
group was assigned a random index date during the index year.

We followed $\mathrm{BC}$ patients and controls up to10 years from the index date. $\mathrm{BC}$ patients and controls who died during the 10-year follow-up period were censored at time of death. There was no loss to follow-up as national registry data covering the whole Danish population were used to assess status at follow-up.

\section{Outcomes and covariates}

We defined CVD as at least one primary or secondary CVD diagnosis in NPR, see Fig. 1. NPR holds information on all patients discharged from Danish hospitals since 1977 and on emergency department and outpatient visits since 1995 [14]. For each hospital contact, one primary and optional secondary diagnoses are registered according to the International Classification of Diseases (ICD). The primary diagnosis is the main reason for the hospital contact and secondary diagnoses identify other relevant diseases.

Furthermore, we identified $\mathrm{BC}$ patients with at least two CVD drug prescriptions (i.e. drugs related to CVD disease management) within the same year in NPRD, see Fig. 2. NPRD includes information on all primary care prescription drugs dispensed from community pharmacies in Denmark coded according to the Anatomical Therapeutic Chemical Classification System (ATC) [16]. We applied the criteria of two prescriptions to exclude persons who were apparently not in continuous treatment. It is not straightforward to identify CVD patients based on NPRD data as the drugs listed in Box 2 are not used exclusively for CVD disease management, and the NPRD does not contain the same information on diagnoses as NPR. However, NPRD data are still relevant to capture the large number of CVD patients who are not registered in NPR because their condition does not require hospitalization or outpatient visits to hospitals.

We used CCI as an aggregate measure of overall comorbidity [19] and diabetes at index date as covariates in multivariate Cox regression analyses. We calculated CCI using information from NPR on primary and secondary diagnoses up to 5 years before the index date. We classified the study population into three groups with a CCI score equal to zero, 1, or 2 and above, respectively. We defined diabetes at index date as at least one primary or secondary diabetes diagnosis in NPR (ICD-10: E10*-E11*) up to 5 years prior to the index date or at least two insulin prescriptions or prescriptions for blood glucose lowering drugs in NPRD (ATC group $A 10 A^{*}$ and $\left.A 10 B^{*}\right)$ within the same year during the 5year period.

We identified persons in the $\mathrm{BC}$ group who had received different types of cancer treatments based on data from NPR. Surgery was defined by a 'surgery procedure code' in combination with $\mathrm{BC}$ diagnosis. Chemotherapy was defined by a BWHA procedure code, radiation therapy was defined by a BWG procedure code, antibody therapy was defined by a $\mathrm{BOHJI}$ procedure code, and hormonal therapy was defined by a BWHC procedure code.

We identified persons in the $\mathrm{BC}$ and control group who died during follow-up (including date of death) based on data from the Civil Registration System.

Data on age, region of residence and education were obtained from the Civil Registration System and education registers.

\section{Statistical analysis}

CVD prevalence rates were calculated as number of persons with at least one CVD primary or secondary

- Heart valve disease (ICD-10: I05.0-I05.1, I34.0-I34.2, I34.9-I35.1, I35.9)

- Hypertension (ICD-10: I10-I15 excl. I11.0, I13.0, I13.2)

- Ischemic heart disease and acute coronary syndrome (ACS)

- Angina pectoris (ICD-10: I20)

- Acute myocardial infarction (ICD-10: I21, I22, I25.2)

- Chronic ischemic heart disease (ICD-10: I25 excl. I25.2)

- Pulmonary heart disease and diseases of pulmonary circulation, including pulmonary embolism, (ICD-10: I26-I28)

- Atrial fibrillation (ICD-10: I48)

- Other cardiac arrhythmias (ICD-10: I49)

- Heart failure including cardiomyopathy and hypotensive heart (and renal) disease with heart (and renal) failure (ICD-10: I50, I42.0, I42.6, I42.7, I42.9, I11.0, I13.0, I13.2)

- Cerebrovascular disease (I60-I69, G45-G46)

- Peripheral vascular disease (I71, I79.0, I73.9, R02, Z95.8, Z95.9))

- Thrombophlebitis and thrombosis (ICD10: I80-I82)

Fig. 1 Types of CVD included in the study (ICD-10 diagnoses) 


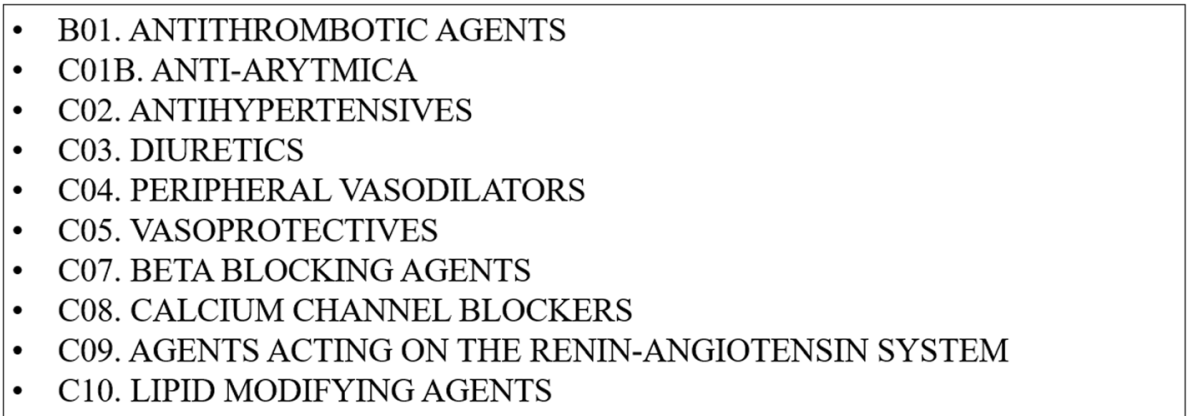

Fig. 2 CVD prescription drugs included in the study (ATC groups)

diagnosis in NPR up to 5 years prior to the index date per 1000 persons for all CVD diagnoses combined and specific types of CVD. Furthermore, number of BC patients and cancer-free controls treated with CVD prescription drugs up to 5 years prior to the index date per 1000 persons were calculated based on NPRD data.

The cumulative incidence of all CVD diagnoses combined as well as specific types of CVD were calculated up to 10 years after the index date (BC diagnosis) allowing for the presence of competing risk of death [20]. A competing risk is an event whose occurrence precludes another event of interest. CVD incidence rates for BC patients and controls were calculated among $\mathrm{BC}$ patients and controls without CVD diagnosis up to 5 years before the index date.

We performed multivariate Cox regression analyses to investigate the risk of developing CVD in $\mathrm{BC}$ patients following chemotherapy, radiation therapy, antibody therapy and hormonal therapy initiated within 9 months after BC diagnosis. The risk of CVD was calculated based on either CVD diagnoses in NPR (model 1) or CVD prescription drugs in NPDR (model 2). Only BC patients without any CVD diagnosis in NPR 5 years prior to the index date were included in model 1 , and only $\mathrm{BC}$ patients without a history of CVD prescription drugs 5 years prior to the index date were included in model 2. We included time dependent exposure dummies that classified the patients' time-to-event' as unexposed until treatment began to avoid immortal time bias [21]. BC patients who had received surgery, but not the cancer treatment in question (chemotherapy, radiation therapy, antibody therapy or hormonal therapy) were used as reference. Adjustments were made for differences between groups with regard to age, region of residence, education and cohort (year of BC diagnosis) as well as comorbidity. Persons who died during follow-up were censored at time of death.

The analyses were performed using Stata 14 (StataCorp, College Station, TX). The statistical significance of differences between groups were evaluated using two- sided t-tests with a $5 \%$ level of significance or $95 \%$ confidence intervals.

There were no missing data except for education. Statistical analyses were carried out using a special category for this group (education unknown).

\section{Results}

The study population included $16,505 \mathrm{BC}$ patients and 165,042 cancer-free controls, see Table 1. By matching design, $\mathrm{BC}$ patients and controls were identical with regard to age, region of residence, and education as well as gender (they were all women).

Of the 16,505 BC patients, 12,715 (77\%) were diagnosed with early stage BC (i.e. stage I-III without distant metastasis) and 997 (6\%) were diagnosed in stage IV (i.e. with distant metastasis), see Table 1 . For the remaining $2793 \mathrm{BC}$ patients (17\%), cancer disease stage at the time of diagnosis was unknown. Almost all $\mathrm{BC}$ patients received surgery $(91 \%), 55 \%$ received radiation therapy, $39 \%$ received hormonal therapy, 33\% received chemotherapy, and $4 \%$ received antibody therapy initiated within 9 months of $\mathrm{BC}$ diagnosis. A larger proportion of $\mathrm{BC}$ patients diagnosed in stage I-III received surgery, radiation therapy and hormonal therapy compared to $\mathrm{BC}$ patients diagnosed in stage IV.

In total, 156 per $1000 \mathrm{BC}$ patients (15.6\%) were registered with at least one CVD diagnosis in hospital records up to 5 years before the index date (BC diagnosis) compared to 151 per 1000 cancer-free controls (15.1\%), see Table 2. The most common type of CVD diagnosis was hypertension $(7.3 \%$ of $\mathrm{BC}$ patients compared to $7.0 \%$ of controls) followed by ischemic heart disease/acute coronary syndrome ( $4.5 \%$ of $\mathrm{BC}$ patient compared to $4.7 \%$ of controls) and cerebrovascular disease $(3.7 \%$ of $\mathrm{BC}$ patients compared to $3.6 \%$ of controls). The prevalence rates of CVD were not significantly different between $\mathrm{BC}$ patients and controls with the exception of atrial fibrillation $(p=0.0019)$, which was higher in $\mathrm{BC}$ patients (3.2\% compared to $2.6 \%$ in controls). 
Table 1 Baseline characteristics of BC patients and cancer-free controls

\begin{tabular}{|c|c|c|c|c|c|}
\hline & \multicolumn{4}{|l|}{$\mathrm{BC}$ patients } & \multirow{2}{*}{$\begin{array}{l}\text { Cancer-free } \\
\text { controls }\end{array}$} \\
\hline & $\begin{array}{l}\text { Stage } \leq \text { III (diagnosed } \\
\text { without distant metastasis) }^{\text {a }}\end{array}$ & $\begin{array}{l}\text { Stage }=\text { IV (diagnosed } \\
\text { with distant metastasis) }\end{array}$ & Stage unknown & Total & \\
\hline & $N=12,715$ & $N=997$ & $N=2793$ & $N=16,505$ & $N=165,042$ \\
\hline \multicolumn{6}{|l|}{ Age group } \\
\hline$<45$ & $11 \%$ & $6 \%$ & $7 \%$ & $10 \%$ & $10 \%$ \\
\hline $45-54$ & $21 \%$ & $12 \%$ & $16 \%$ & $20 \%$ & $20 \%$ \\
\hline $55-64$ & $31 \%$ & $25 \%$ & $23 \%$ & $29 \%$ & $29 \%$ \\
\hline $65-74$ & $21 \%$ & $27 \%$ & $19 \%$ & $21 \%$ & $21 \%$ \\
\hline $75+$ & $15 \%$ & $29 \%$ & $35 \%$ & $19 \%$ & $19 \%$ \\
\hline \multicolumn{6}{|l|}{ Region of residence } \\
\hline Northern Jutland & $10 \%$ & $10 \%$ & $10 \%$ & $10 \%$ & $10 \%$ \\
\hline Central Jutland & $21 \%$ & $22 \%$ & $19 \%$ & $20 \%$ & $20 \%$ \\
\hline Southern Denmark & $23 \%$ & $19 \%$ & $26 \%$ & $23 \%$ & $23 \%$ \\
\hline Capital & $30 \%$ & $29 \%$ & $31 \%$ & $30 \%$ & $30 \%$ \\
\hline Zealand & $16 \%$ & $20 \%$ & $15 \%$ & $16 \%$ & $16 \%$ \\
\hline \multicolumn{6}{|l|}{ Education } \\
\hline Low & $38 \%$ & $49 \%$ & $37 \%$ & $38 \%$ & $38 \%$ \\
\hline Medium & $39 \%$ & $30 \%$ & $32 \%$ & $37 \%$ & $37 \%$ \\
\hline High & $19 \%$ & $11 \%$ & $14 \%$ & $18 \%$ & $18 \%$ \\
\hline Unknown & $4 \%$ & $10 \%$ & $17 \%$ & $6 \%$ & $6 \%$ \\
\hline \multicolumn{6}{|c|}{ Cancer treatment started within 9 months from BC diagnosis } \\
\hline Surgery & $96 \%$ & $63 \%$ & $81 \%$ & $91 \%$ & N.a. \\
\hline Chemotherapy & $35 \%$ & $37 \%$ & $22 \%$ & $33 \%$ & N.a. \\
\hline Radiation therapy & $62 \%$ & $28 \%$ & $34 \%$ & $55 \%$ & N.a. \\
\hline Antibody therapy & $4 \%$ & $7 \%$ & $2 \%$ & $4 \%$ & N.a. \\
\hline Hormonal therapy & $41 \%$ & $38 \%$ & $26 \%$ & $39 \%$ & N.a. \\
\hline
\end{tabular}

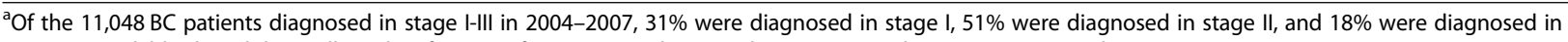
stage III. Available data did not allow classification of BC patients diagnosed in 2003 according to stage I, II and III

In total, 447 per $1000 \mathrm{BC}$ patients (44.7\%) had a history of CVD drug prescriptions (i.e. drugs related to CVD disease management) when diagnosed with BC compared to 445 per 1000 cancer-free controls (44.5\%), see Additional file 1. Diuretics were the most common type of drug used by both $\mathrm{BC}$ patients and controls (20\%) followed by agents acting on the renin-angiotensin system (14\%) and beta blocking agents (11\%). There were no significant differences between $\mathrm{BC}$ patients and controls with regards to these types of CVD drug prescriptions. However, more controls than $\mathrm{BC}$ patients had been treated with antithrombotic agents $(11 \%$ versus $10 \%, p<0.01)$ and lipid modifying agents (7\% versus $6 \%, p<0.001$ ), see Supplementary Table 1.
Up to approximately 6 years after the index date, the cumulative incidence of all CVD diagnoses combined was higher in $\mathrm{BC}$ patients than in cancer-free controls taking account of the presence of competing risk of death, see Fig. 3. Hereafter, the difference in cumulative CVD incidence between $\mathrm{BC}$ patients and controls was no longer statistically significant. After 10 years, $28 \%$ of both $\mathrm{BC}$ patients and controls (without any CVD diagnosis in NPR up to 5 years before the index date) had at least one CVD diagnosis in NPR. The most common types of CVD diagnosis were hypertension with a cumulative incidence of $15 \%$ after 10 years followed by ischemic heart disease/acute coronary syndrome, cerebrovascular disease and atrial fibrillation (all with a cumulative incidence of $5 \%$ after 10 years). 
Table 2 Number of persons with CVD diagnosis before the index date per 1000 persons

\begin{tabular}{lllll}
\hline & BC patients & Cancer-free controls & Difference & P value \\
\hline CVD & $\mathbf{1 5 6 . 3}$ & $\mathbf{1 5 0 . 7}$ & $\mathbf{5 . 6}$ & $\mathbf{0 . 0 5 4 6}$ \\
Hypertension & 73.4 & 69.6 & 3.8 & 0.0687 \\
Ischemic heart disease/ACS & 44.9 & 47.3 & -2.4 & 0.1659 \\
Cerebrovascular disease & 36.5 & 36.4 & 0.1 & 0.9409 \\
Atrial fibrillation & 30.2 & 26.1 & 4.1 & 0.0019 \\
Heart failure & 21.4 & 20.1 & 1.4 & 0.0 \\
Heart valve disease & 9.0 & 9.1 & 0.2251 \\
Thrombophlebitis/thrombosis & 8.5 & 7.6 & 0.9 & 0.9745 \\
Other cardiac arrhythmias & 8.2 & 7.3 & 0.9 & 0.2017 \\
Peripheral vascular disease & 6.7 & 7.7 & -1.1 & 0.2189 \\
Pulmonary heart disease & 4.7 & 4.0 & 0.7 & 0.1272 \\
\hline
\end{tabular}

During the first 2 years after the index date, the cumulative incidence of most types of CVD was statistically significantly higher in $\mathrm{BC}$ patients than controls, see Fig. 4. However, only the cumulative incidence of heart failure, thrombophlebitis/thrombosis and pulmonary heart disease including pulmonary embolism remained higher in $\mathrm{BC}$ patients during the entire 10-year followup period. After 10 years, $2.7 \%$ of $\mathrm{BC}$ patients compared to $2.5 \%$ of controls had developed heart failure, $2.7 \%$ of $\mathrm{BC}$ patients compared to $1.5 \%$ of controls had developed thrombophlebitis/thrombosis, and $1.5 \%$ of $\mathrm{BC}$ patients compared to $1.0 \%$ of controls had developed pulmonary heart disease.

The risk of CVD was significantly elevated in BC patients after chemotherapy $(\mathrm{HR}>1)$, no matter whether
CVD was defined according to CVD diagnoses registered in NPR (95\% CI: 1.030-1.353), see Table 3 (model 1), or CVD drug prescriptions registered in NPRD (95\% CI: 1.226-1.520), see Table 3 (model 2). Furthermore, the risk of CVD was significantly higher after radiation therapy (HR $>1$ ), when CVD was defined according to CVD drug prescriptions in NPDR (95\% CI: 1.023-1.251), but not CVD diagnoses in NPR (95\% CI: 0.887-1.117). There were no statistically significant results regarding the risk of CVD after hormonal or antibody therapy.

When investigating the risk of different types of CVD after chemotherapy, we found that the risk of heart failure and thrombophlebitis/thrombosis was significantly elevated in $\mathrm{BC}$ patients who received chemotherapy compared to $\mathrm{BC}$ patients who did not, see Table 4.

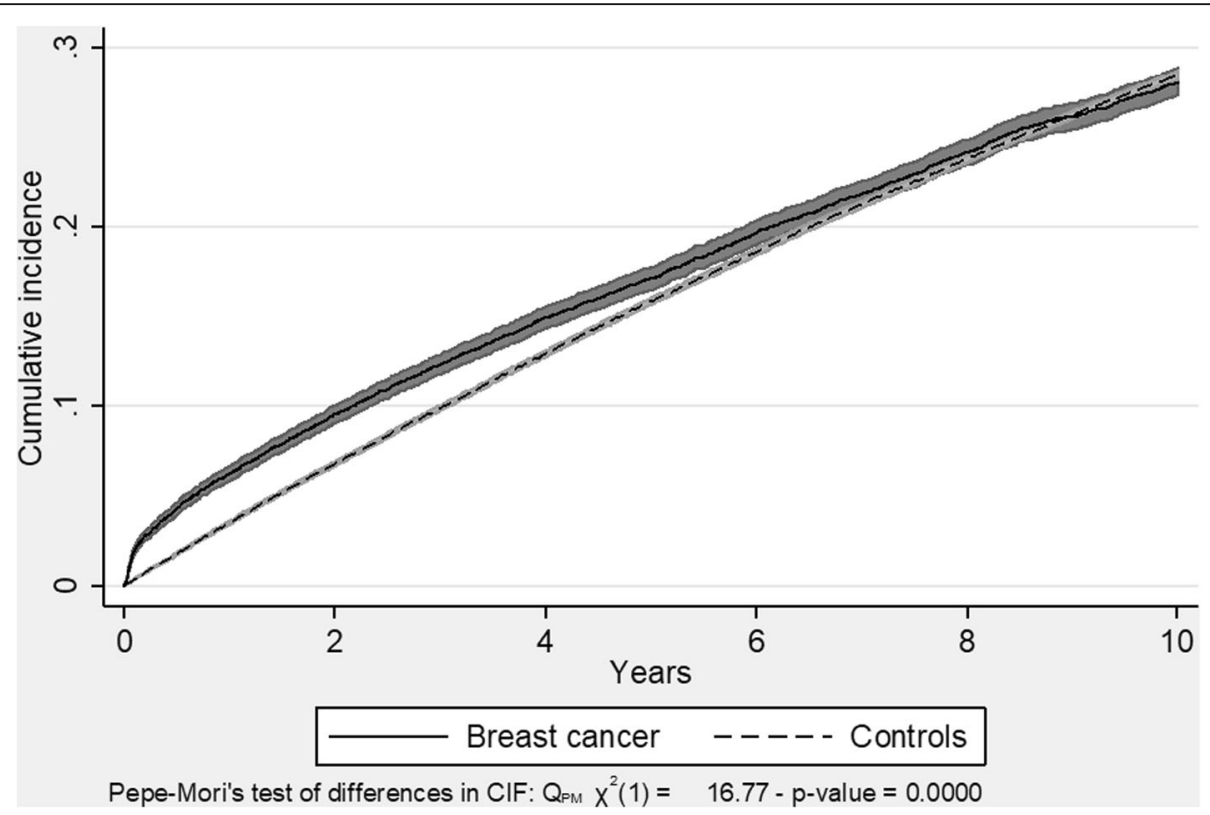

Fig. 3 Cumulative incidence of all CVD diagnoses combined, proportion with at least one CVD diagnosis 

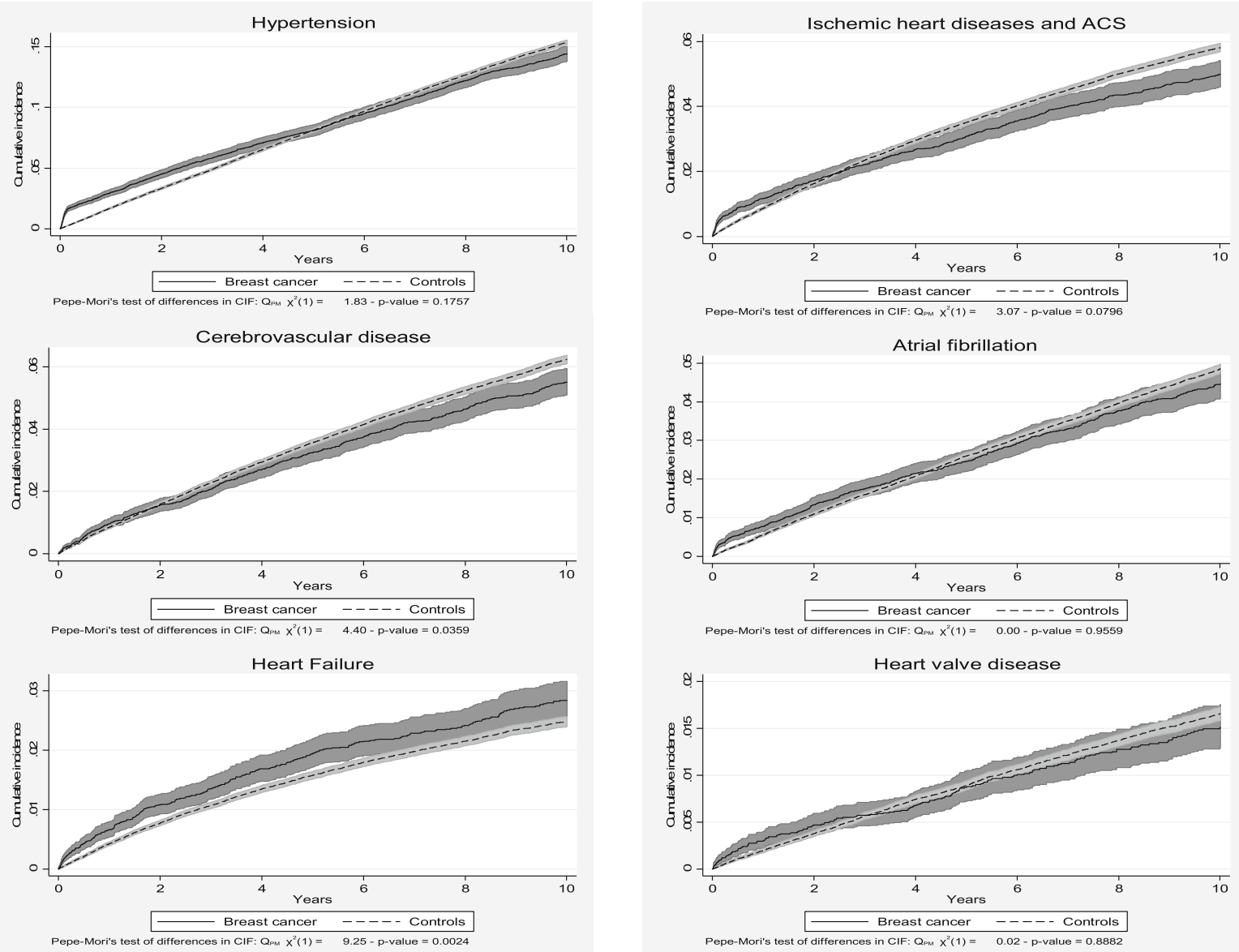

Heart valve disease

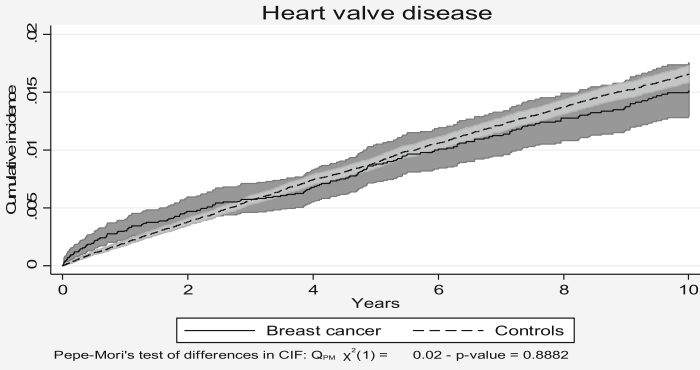

Thrombophlebitis/thrombosis

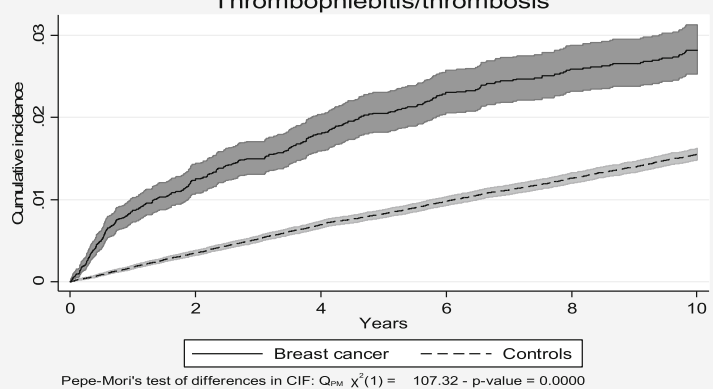

Other cardiac arrhythmies
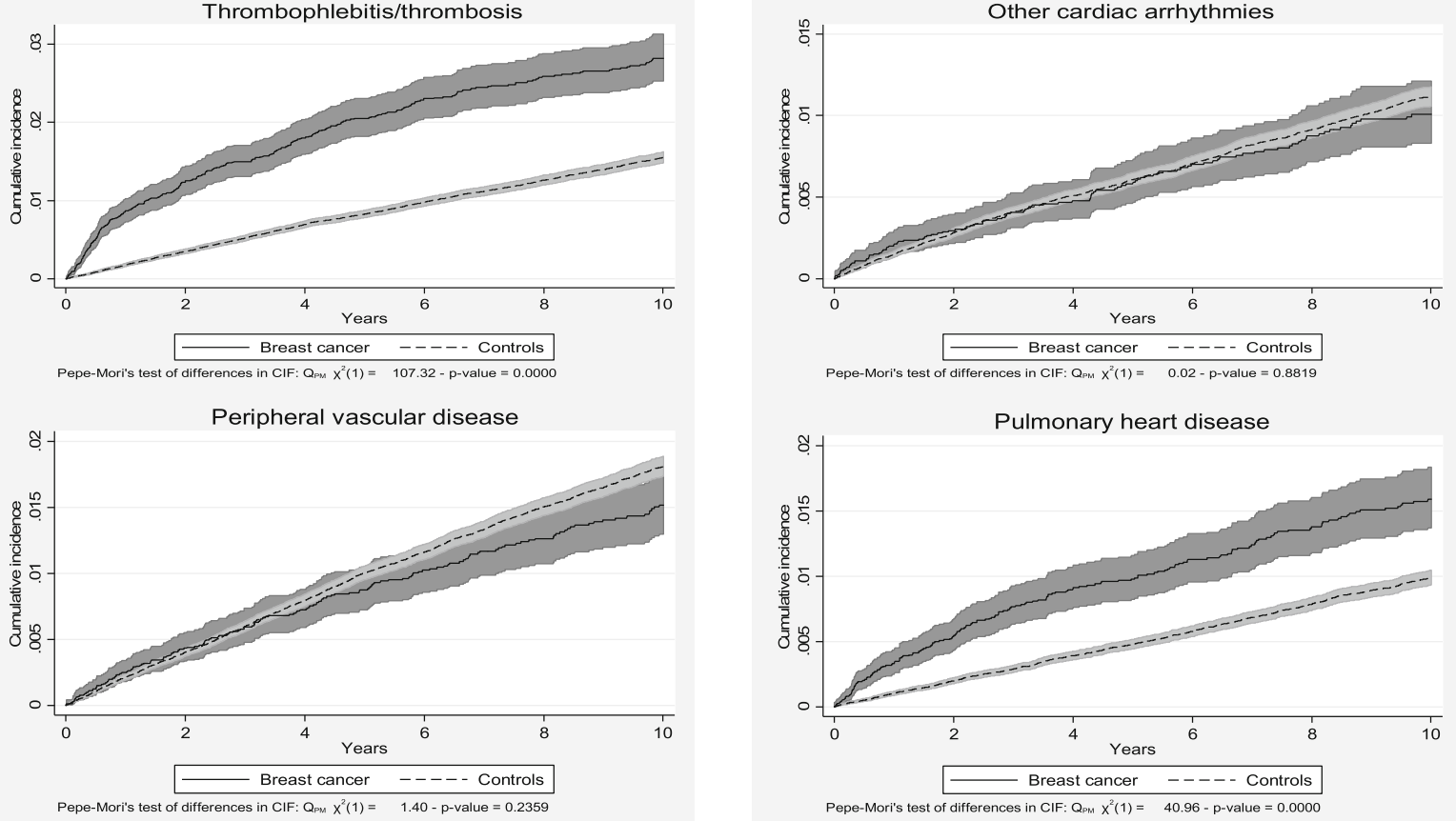

Fig. 4 Cumulative incidence of specific types of CVD diagnoses, proportion with CVD diagnosis 
Table 3 Hazard ratio (HR) of CVD in BC patients after different types of cancer treatment

\begin{tabular}{lll}
\hline & $\begin{array}{l}\text { Model 1: } \\
\text { CVD diagnosis in NPR } \\
{[95 \% \text { Cl] }}\end{array}$ & $\begin{array}{l}\text { Model 2: } \\
\text { CVD drug prescriptions in NPDR } \\
\text { [95\% Cl] }\end{array}$ \\
\hline Chemotherapy & $1.180^{*}$ & $1.365^{* * *}$ \\
{$[1.226 ; 1.520]$} & $1.131^{*}$ \\
Radiation therapy & {$[1.030 ; 1.353]$} & {$[1.023 ; 1.251]$} \\
Antibody therapy & 0.996 & 1.032 \\
& {$[0.887 ; 1.117]$} & {$[0.827 ; 1.290]$} \\
Hormonal therapy & 1.029 & 0.983 \\
No surgery & {$[0.759 ; 1.394]$} & {$[0.832 ; 1.162]$} \\
Distant metastasis when diagnosed with BC & 0.834 & 1.091 \\
& {$[0.686 ; 1.014]$} & {$[0.914 ; 1.301]$} \\
Number of observations, N & 1.112 & $1.329^{*}$
\end{tabular}

95\% confidence intervals in brackets. The following covariates were included in the models: Age, region of residence, education, cohort (year of diagnosis) and comorbidity at index date (CCl, diabetes or CVD (CVD at index date was defined by either CVD drug prescriptions in NPDR (model 1) or CVD diagnoses in NPR (model 2)). Comorbidity at index date was associated with an increased risk of CVD during the follow-up period no matter whether comorbidity was measured by $\mathrm{CCl}$, diabetes or CVD

${ }^{*} p<0.05,{ }^{* *} p<0.01,{ }^{* * *} p<0.001$

Furthermore, we found that the differences in risk of developing heart failure and thrombophlebitis/thrombosis increased during the 10-year follow-up period, see Fig. 5.

\section{Discussion}

We found that $15.6 \%$ of $\mathrm{BC}$ patients were registered with at least one CVD diagnosis in hospital records up to 5 years before $\mathrm{BC}$ diagnosis, and a considerably higher percentage of patients had a history of CVD drug prescriptions (i.e. drugs related to CVD disease management). Overall, BC patients and cancer-free controls were similar with regard to CVD comorbidity at index date. The most common types of CVD diagnoses in both $\mathrm{BC}$ patients and controls were hypertension, ischemic heart disease/acute coronary syndrome, and cerebrovascular disease.

After BC diagnosis, the incidence of CVD was significantly higher in $\mathrm{BC}$ patients than controls for all CVD diagnoses combined up to approximately 6 years after the index date ( $\mathrm{BC}$ diagnosis) taking account of the presence of competing risk of death. Higher incidence of CVD in BC patients may be related to cancer treatments such as anthracycline chemotherapy, HER2-targeted agents, aromatase inhibitors, and radiation therapy. It may also be related to lifestyle factors such as obesity and sedentary behaviour if $\mathrm{BC}$ patients are more exposed to these risk factors than controls on average.

However, $28 \%$ of both BC patients and controls (without any CVD diagnosis in NPR 5 years before the index date) had at least one CVD diagnosis in NPR 10 years after the index date. Only the cumulative incidence of heart failure, thrombophlebitis/thrombosis and pulmonary heart disease including pulmonary embolism remained higher in $\mathrm{BC}$ patients than controls during the entire 10-year follow-up period. Furthermore, the risk of heart failure and thrombophlebitis/thrombosis was significantly higher in $\mathrm{BC}$ patients who had received chemotherapy compared to $\mathrm{BC}$ patients who had not as well as cancer-free controls. Heart failure has been associated with chemotherapy in previous studies [22], and it is well established that cancer patients have increased risk of thrombotic complications including e.g. deep vein thrombosis, pulmonary embolism and arterial thrombosis [23].

Contrary to our results, Abel-Qadir et al. [9] found that the prevalence of different types of CVD was significantly higher in $\mathrm{BC}$ patients compared to cancer-free controls. A possible explanation for this discrepancy could be that Abel-Qadir et al. used different algorithms to determine the presence of CVD preceding the index date. Other possible explanations are differences in the definition of the study population and matching criteria, as Abel-Qadir et al. included women diagnosed with early stage $\mathrm{BC}$ only, and controls were matched to $\mathrm{BC}$ patients according to gender and age, but not education as in our study.

Abel-Qadir et al. [9] found a significantly higher incidence of CVD hospitalizations due to heart failure, cerebrovascular disease and arrhythmias in BC patients compared to cancer-free controls whereas we did not 


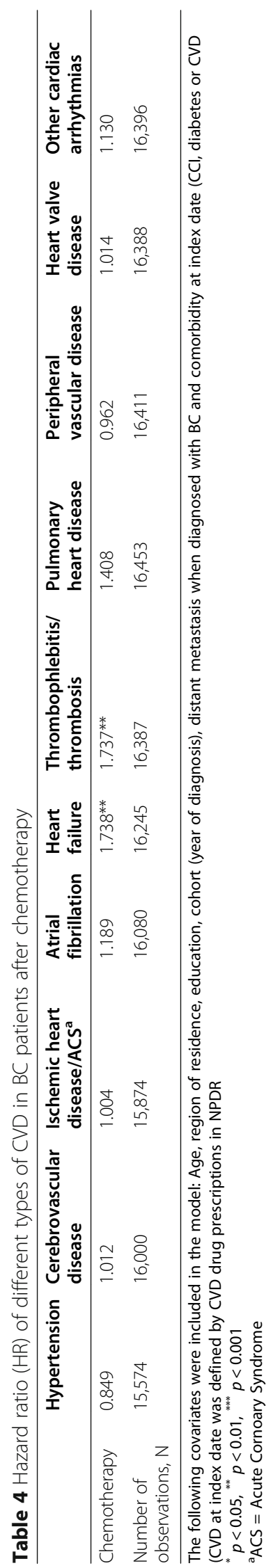



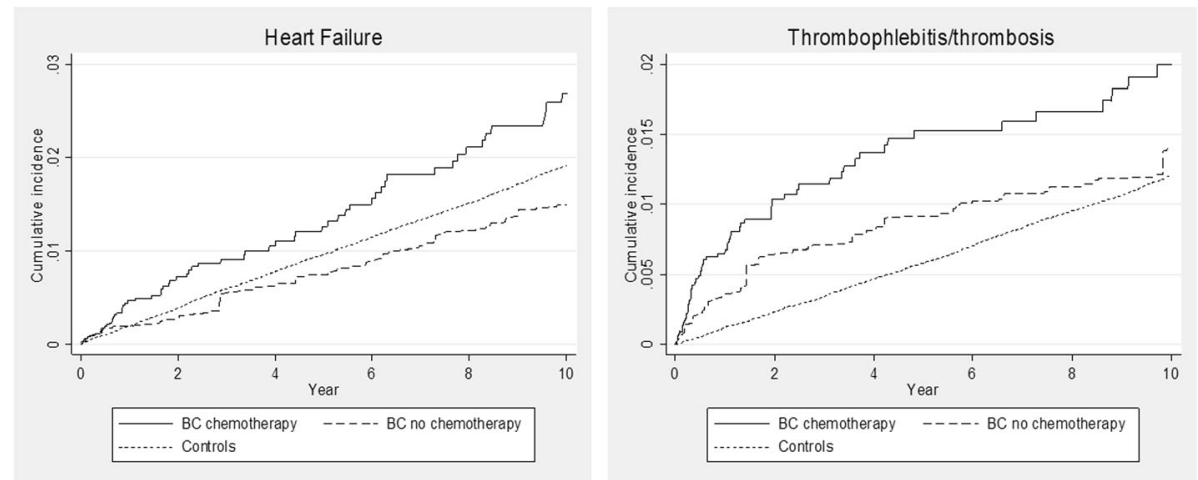

Fig. 5 Cumulative incidence of heart failure and thrombophlebitis/thrombosis after chemotherapy, proportion with diagnosis

find this for cerebrovascular disease or arrhythmias. In accordance with our results, Strongman et al. [10] found a statistically significantly higher incidence of heart failure and venous thromboembolism in BC patients compared to cancer-free controls, but not significant differences in the incidence of arrhythmia, stroke or peripheral vascular disease. Furthermore, Strongman et al. found that the incidence of coronary artery disease was significantly lower in $\mathrm{BC}$ patients compared to controls.

Existing studies have shown an elevated risk of CVD in BC patients after anthracycline-based chemotherapy, radiation therapy and antibody therapy [9, 24-26]. Our study confirms an elevated risk of CVD after chemotherapy. Unfortunately, data available for our study did not allow distinction between different types of chemotherapy. We also saw a tendency towards a higher risk of CVD after radiation and antibody therapy, but these results were in general not statistically significant.

Hormonal agents like tamoxifen approved for BC treatment more than 30 years ago can have both beneficial and detrimental effects on the cardiovascular system [22]. Studies have shown that tamoxifen has a protective effect on lipid metabolism [27-29], but at the same time increases the risk of venous thrombosis and thromboembolism [30]. In our study, we did not find a higher risk of CVD after hormonal therapy, but we saw a higher incidence of thrombotic complications in $\mathrm{BC}$ patients compared to cancer-free controls.

The present study has several strengths. Firstly, it is a study at the population level based on a large real-world dataset with no selection bias or other potential issues related to more segmented and selective populations. Secondly, the study population was identified from CAR, which has high completeness and validity due to use of notifications from different data sources and quality control [12]. Thirdly, we used prescription data from NPRD to identify CVD patients as a supplement to data from $\mathrm{NPR}$, as the latter registry does not include patients who require primary care only. NPRD is the national registry of prescription drugs dispensed from community pharmacies and is considered both complete and valid from 1995 and onwards [16]. Fourthly, the study included a cancer-free control group, and the use of exact matching ensured that $\mathrm{BC}$ patients and controls were comparable with regard to age, region of residence, and education as well as gender (they were all women). We did not include CCI as a matching criteria to avoid overmatching, and we preferred exact matching to propensity score matching because exact matching ensures that groups are identical with regard to the matching criteria used. Finally, we allowed for the presence of competing risk of death when estimating the cumulative incidence function $[20,31]$. Since BC patients had a higher risk of dying during the 10-year follow-up period than controls, ignoring the presence of competing risk could result in substantial bias.

The study also has limitations. Firstly, the study relies on diagnosis and procedure codes in NPR, which may contain errors or be incomplete, e.g. many doctors code the primary diagnosis only, even if the patient has other relevant diseases [32]. Secondly, NPR does not contain information on patients who require primary care only as mentioned above, and it is not straightforward to identify CVD patients based on NPRD data as prescription drugs used for CVD disease management may have other indications. Furthermore, some patients are not diagnosed and consequently not included in NPR or NPRD. Thirdly, drug adherence cannot be ascertained based on NPRD data. If CVD drug adherence is lower in $\mathrm{BC}$ patients compared to cancer-free controls, this may be an area of intervention to reduce the risk of serious CVD events in $\mathrm{BC}$ patients, but we have no reason to suspect this. Fourthly, as our study population is relatively homogeneous with regard to race, our results may be less generalizable to more diverse patient populations, especially given higher rates of CVD risk factors in some racial minorities. Still, the patient population in Denmark is comparable to most Northern European 
countries. Finally, there is a risk of confounding due to the observational nature of the study. The risk of confounding related to observable baseline characteristics was minimised by exact matching and a multivariable regression design, but $\mathrm{BC}$ patients and controls may differ on non-observable lifestyle factors, that influence the risk of CVD. However, we expect lifestyle factors to be similar in BC patients and controls because education was included as a matching criteria in our study and is considered to be a proxy for lifestyle. Similar comorbidity at index date also indicates a comparable lifestyle between $\mathrm{BC}$ patients and controls.

\section{Conclusions}

In conclusion, we found that $15.6 \%$ of $\mathrm{BC}$ patients were registered with at least one CVD diagnosis in hospital records when diagnosed with $\mathrm{BC}$. Overall, $\mathrm{BC}$ patients and controls were similar with regard to CVD comorbidity before $\mathrm{BC}$ diagnosis. After $\mathrm{BC}$ diagnosis, the incidence of all CVD diagnoses combined was significantly higher in $\mathrm{BC}$ patients than controls up to approximately 6 years after the index date. The incidence of heart failure, thrombophlebitis/thrombosis and pulmonary heart disease including pulmonary embolism remained higher in $\mathrm{BC}$ patients than controls during the entire 10-year follow-up period. Our study supports the need for strategies and novel approaches to minimise and manage the risk of CVD in BC patients. Special attention should be given to reduce the risk of heart failure, thrombophlebitis/thrombosis and pulmonary heart disease including pulmonary embolism in $\mathrm{BC}$ patients in the future.

\section{Abbreviations}

ATC: Anatomical Therapeutic Chemical Classification System; BC: Breast cancer; CAR: The Danish Cancer Registry; CCl: Charlson Comorbidity Index; 95\% Cl: 95\% confidence interval; CVD: Cardiovascular disease; HR: Hazard ratio; ICD: International Classification of Diseases; NPRD: Danish Prescription Drug Registry; NPR: the Danish National Patient Registry

\section{Supplementary Information}

The online version contains supplementary material available at https://doi. org/10.1186/s12885-021-08716-5.

Additional file 1: Table S1. Number of persons with CVD drug prescription before the index date per 1000 persons.

\section{Acknowledgements}

Not applicable.

\section{Authors' contributions}

MJ, CK, MSJ, PBP, HK, TK and MA were all involved in the conception and design of the study. CK and MSJ had the main responsibility for carrying out the statistical analyses, and MJ had the main responsibility for drafting the article. All authors were involved in the analysis and interpretation of data, revised the article critically and approved the final version for publication.

\section{Funding}

The study was funded by Pfizer Denmark. A Study Steering Committee consisting of experts in oncology and cardiology, employees from VIVE and
Pfizer Denmark (the authors) designed the study and gave feedback on the collection, analysis and interpretation of data, as well as the writing of the manuscript.

\section{Availability of data and materials}

The data that support the findings of this study are available from the Danish Health Data Authority and Statistics Denmark but restrictions apply to the availability of these data, which were used under license for the current study, and so are not publicly available.

\section{Declarations}

Ethics approval and consent to participate

Neither informed consent from patients nor approval from an ethics committee was required by Danish law since the study was based on retrospective registry data only. Research Ethics Committees in Denmark assess only biomedical research.

Administrative permissions to access the raw data used in the study were acquired from Statistics Denmark and The Danish Health Data Authority. The study was carried out in accordance with international ethical standards including the EU general data protection regulation. Data for statistical analyses were anonymized, and study results were reported so that it is not possible to identify individual persons.

\section{Consent for publication}

Not applicable.

\section{Competing interests}

MJ, CK and MSJ are employees of the National Center for Social Science Research (VIVE), which was a paid vendor to Pfizer Denmark on the project. VIVE is an independent research institute, which is under obligation by law to disseminate the results of its work to relevant public and private stakeholders and the public in general.

MA and TK are medical doctors with expertise in breast cancer and cardiovascular disease, respectively, and both report personal fees from Pfizer Denmark during the conduct of the study.

PBP and HK are employees of Pfizer Denmark and own shares in Pfizer Inc. outside submitted work.

\section{Author details}

'VIVE, The Danish Center for Social Science Research, Herluf Trolles Gade 11, DK-1152 Copenhagen K, Denmark. VIVE, The Danish Center for Social Science Research, Oluf Palmes Allé 22, DK-8200 Aarhus N, Denmark. ${ }^{3}$ Pfizer Denmark, Lautrupvang 8, DK-2750 Ballerup, Denmark. ${ }^{4}$ Department of Cardiology, Herlev-Gentofte University Hospital, Borgmester lb Juuls Vej 1, DK-2730 Herlev, Denmark. ${ }^{5}$ Department of Oncology, Copenhagen University Hospital, Rigshospitalet, Blegdamsvej 9, DK-2100 Copenhagen Oe, Denmark.

Received: 11 November 2020 Accepted: 23 August 2021

Published online: 18 September 2021

References

1. Jorgensen TL, Hallas J, Friis S, Herrstedt J. Comorbidity in elderly cancer patients in relation to overall and cancer-specific mortality. Br J Cancer. 2012;106(7):1353-60. https://doi.org/10.1038/bjc.2012.46.

2. Søgaard M, Thomsen RW, Bossen KS, Sørensen HT, Nørgaard M. The impact of comorbidity on cancer survival: a review. Clin Epidemiol. 2013;5(Suppl 1): 3-29. https://doi.org/10.2147/CLEP.S47150.

3. Statens Serum Institut. Sammenhæng mellem komorbiditet og behandling inden for standardforløbstiderne i pakkeforløb for kræft. København: Statens Serum Institut \& National Sundhedsdokumentation og -IT; 2015.

4. Land LH, Dalton SO, Jensen MB, Ewertz M. Impact of comorbidity on mortality: a cohort study of 62,591 Danish women diagnosed with early breast cancer, 1990-2008. Breast Cancer Res Treat. 2012;131(3):1013-20. https://doi.org/10.1007/s10549-011-1819-1.

5. Berglund A, Wigertz A, Adolfsson J, Ahlgren J, Fornander T, Warnberg F, et al. Impact of comorbidity on management and mortality in women diagnosed with breast cancer. Breast Cancer Res Treat. 2012;135(1):281-9. https://doi.org/10.1007/s10549-012-2176-4.

6. O'Connor T, Edge S, Kossoff E, Groman A, Wilding G, Ademiyiwa F, et al. Factors affecting the delivery of adjuvant/neoadjuvant chemotherapy in 
older women with breast cancer. J Geriatr Oncol. 2012;3(4):320-8. https:// doi.org/10.1016/j.jgo.2012.06.006.

7. Punglia RS, Saito AM, Neville BA, Earle CC, Weeks JC. Impact of interval from breast conserving surgery to radiotherapy on local recurrence in older women with breast cancer: retrospective cohort analysis. BMJ. 2010; 340(mar02 2):c845. https://doi.org/10.1136/bmj.c845.

8. Ewertz M, Land LH, Dalton SO, Cronin-Fenton D, Jensen MB. Influence of specific comorbidities on survival after early-stage breast cancer. Acta Oncol. 2018;57(1):129-34. https://doi.org/10.1080/0284186X.2017.1407496.

9. Abdel-Qadir H, Thavendiranathan P, Austin PC, Lee DS, Amir E, Tu JV, et al. The risk of heart failure and other cardiovascular hospitalizations after early stage breast cancer: a matched cohort study. J Natl Cancer Inst. 2019;111(8): 854-62. https://doi.org/10.1093/jnci/djy218.

10. Strongman H, Gadd S, Matthews A, Mansfield KE, Stanway S, Lyon AR, et al. Medium and long-term risks of specific cardiovascular diseases in survivors of 20 adult cancers: a population-based cohort study using multiple linked UK electronic health records databases. Lancet. 2019;394(10203):1041-54. https://doi.org/10.1016/50140-6736(19)31674-5.

11. Herrett E, Gallagher AM, Bhaskaran K, Forbes H, Mathur R, van Staa T, et al. Data resource profile: clinical practice research datalink (CPRD). Int J Epidemiol. 2015;44(3):827-36. https://doi.org/10.1093/ije/dyv098.

12. Gjerstorff ML. The Danish cancer registry. Scand J Public Health. 2011; 39(Suppl 7):42-5. https://doi.org/10.1177/1403494810393562.

13. Lynge E, Sandegaard JL, Rebolj M. The Danish national patient register. Scand J Public Health. 2011;39(7 Suppl):30-3. https://doi.org/10.1177/14034 94811401482

14. Schmidt M, Schmidt SA, Sandegaard JL, Ehrenstein V, Pedersen L, Sorensen HT. The Danish National Patient Registry: a review of content, data quality, and research potential. Clin Epidemiol. 2015;7:449-90. https://doi.org/10.214 7/CLEP.S91125.

15. Kildemoes HW, Sorensen HT, Hallas J. The Danish national prescription registry. Scand J Public Health. 2011;39(7 Suppl):38-41. https://doi.org/10.11 77/1403494810394717.

16. Pottegard A, Schmidt SAJ, Wallach-Kildemoes H, Sorensen HT, Hallas J, Schmidt M. Data resource profile: the Danish national prescription registry. Int J Epidemiol. 2017:46(3):798-798f

17. Jensen VM, Rasmussen AW. Danish education registers. Scand J Public Health. 2011;39(7 Suppl):91-4. https://doi.org/10.1177/1403494810394715.

18. Pedersen CB. The Danish civil registration system. Scand J Public Health. 2011;39(7 Suppl):22-5. https://doi.org/10.1177/1403494810387965.

19. Charlson ME, Pompei P, Ales KL, MacKenzie CR. A new method of classifying prognostic comorbidity in longitudinal studies: development and validation. J Chronic Dis. 1987;40(5):373-83. https://doi.org/10.1016/0021-9681(87)90171-8.

20. Austin PC, Lee DS, Fine JP. Introduction to the analysis of survival data in the presence of competing risks. Circ: Cardiovasc Qual Outcomes. 2016; 133(6):601-9.

21. Suissa S. Immortal time bias in observational studies of drug effects. Pharmacoepidemiol Drug Saf. 2007;16(3):241-9. https://doi.org/10.1002/ pds.1357.

22. Mehta LS, Watson KE, Barac A, Beckie TM, Bittner V, Cruz-Flores S, et al. Cardiovascular disease and breast cancer: where these entities intersect: a scientific statement from the American Heart Association. Circulation. 2018; 137(8):e30-66.

23. Elyamany G, Alzahrani AM, Bukhary E. Cancer-associated thrombosis: an overview. Clin Med Insights Oncol. 2014;8:129-37. https://doi.org/10.4137/ CMO.S18991.

24. Darby SC, Ewertz M, McGale P, Bennet AM, Blom-Goldman U, Brønnum D, et al. Risk of ischemic heart disease in women after radiotherapy for breast cancer. $\mathrm{N}$ Engl J Med. 2013;368(11):987-98. https://doi.org/10.1056/NEJMoa1209825.

25. McGale P, Darby SC, Hall P, Adolfsson J, Bengtsson NO, Bennet AM, et al. Incidence of heart disease in 35,000 women treated with radiotherapy for breast cancer in Denmark and Sweden. Radiother Oncol. 2011;100(2):16775. https://doi.org/10.1016/j.radonc.2011.06.016.

26. Rehammar JC, Jensen MB, McGale P, Lorenzen EL, Taylor C, Darby SC, et al. Risk of heart disease in relation to radiotherapy and chemotherapy with anthracyclines among 19,464 breast cancer patients in Denmark, 1977-2005. Radiother Oncol. 2017;123(2):299-305. https://doi.org/10.1016/j.radonc.2017. 03.012.

27. Dewar JA, Horobin JM, Preece PE, Tavendale R, Tunstall-Pedoe H, Wood RA. Long term effects of tamoxifen on blood lipid values in breast cancer. BMJ. 1992;305(6847):225-6. https://doi.org/10.1136/bmj.305.6847.225.
28. Grey AB, Stapleton JP, Evans MC, Reid IR. The effect of the anti-estrogen tamoxifen on cardiovascular risk factors in normal postmenopausal women. J Clin Endocrinol Metab. 1995;80(11):3191-5. https://doi.org/10.1210/jcem. 80.11 .7593425$.

29. Esteva FJ, Hortobagyi GN. Comparative assessment of lipid effects of endocrine therapy for breast cancer: implications for cardiovascular disease prevention in postmenopausal women. Breast. 2006;15(3):301-12. https:// doi.org/10.1016/j.breast.2005.08.033.

30. Saphner T, Tormey DC, Gray R. Venous and arterial thrombosis in patients who received adjuvant therapy for breast cancer. J Clin Oncol. 1991;9(2): 286-94. https://doi.org/10.1200/JCO.1991.9.2.286.

31. Abdel-Qadir H, Fang J, Lee DS, Tu JV, Amir E, Austin PC, et al. Importance of considering competing risks in time-to-event analyses: application to stroke risk in a retrospective cohort study of elderly patients with atrial fibrillation. Circul: Cardiovasc Qual Outcomes. 2018:11(7):1-11.

32. Kumler T, Gislason GH, Kirk V, Bay M, Nielsen OW, Kober L, et al. Accuracy of a heart failure diagnosis in administrative registers. Eur J Heart Fail. 2008; 10(7):658-60. https://doi.org/10.1016/j.ejheart.2008.05.006.

\section{Publisher's Note}

Springer Nature remains neutral with regard to jurisdictional claims in published maps and institutional affiliations.
Ready to submit your research? Choose BMC and benefit from:

- fast, convenient online submission

- thorough peer review by experienced researchers in your field

- rapid publication on acceptance

- support for research data, including large and complex data types

- gold Open Access which fosters wider collaboration and increased citations

- maximum visibility for your research: over $100 \mathrm{M}$ website views per year

At BMC, research is always in progress.

Learn more biomedcentral.com/submissions 